\title{
DYNAMICS OF LARGE-SCALE MAGNETIC FIELDS
}

\author{
G. V. KUKLIN
}

Siberian Institute of Terrestrial Magnetism, Ionosphere and Radio Propagation, Irkutsk, U.S.S.R.

\begin{abstract}
A discussion is given of the dynamics of large-scale solar magnetic fields as a joint action of a transport by a regular velocity field, by a generalized diffusion, by coming to the surface and sinking and by sources of origin and destruction of magnetic fields. Also these processes are discussed using the observed picture of magnetic field dynamics.
\end{abstract}

The evolution and dynamics of the large-scale weak solar magnetic fields studied first by Bumba and Howard (1965) are not clear, although investigations of such kind by many authors are in progress now. Only the structure of these large-scale magnetic fields and their connection with other solar phenomena were studied relatively in detail. Because the large-scale magnetic fields are closely connected with the interplanetary magnetic field and with the theory of solar activity, some formal considerations may be of interest.

Let us consider the simplest form of the fundamental equation of hydromagnetics describing the magnetic field evolution in the case of the Sun.

$$
\frac{\partial \mathbf{H}}{\partial t}=\operatorname{rot}[\mathbf{V} \times \mathbf{H}]-\operatorname{rot}\left(v_{m} \operatorname{rot} \mathbf{H}\right) \text {. }
$$

This equation for the $z$-component of the magnetic field may be written in such a way

$$
\begin{aligned}
\frac{\partial \mathbf{H}_{z}}{\partial t}= & -(\mathbf{V} \nabla) H_{z}-H_{z} \operatorname{div} \mathbf{V}+v_{m} \Delta H_{z}+\nabla v_{m} \nabla H_{z}+ \\
& +v_{m} \frac{\partial^{2} H_{z}}{\partial z^{2}}-V_{z} \frac{\partial H_{z}}{\partial z}+\mathbf{h} \nabla V_{z}-\nabla v_{m} \frac{\partial \mathbf{h}}{\partial z}
\end{aligned}
$$

where $V$ and $h$ are the transverse velocity and magnetic fields and $\operatorname{div}, \nabla$ and $\Delta$ are the differential operators in the $X O Y$ plane. If we identify $H_{z}$ with the longitudinal component of the magnetic field $H_{\|}$observed at a solar surface element which is approximately normal to the sight line, then the above equation of the magnetic field dynamics may be interpreted in the following way.

The term $v_{m} \Delta H_{z}$ describes the ohmic dissipation of the longitudinal magnetic field $H_{z}$ in the $X O Y$ plane. The term $\nabla v_{m} \nabla H_{z}$ takes into consideration the changes of $v_{m}$ along the $X O Y$ plane. The term $-(\mathrm{V} \nabla) H_{z}$ is connected with the transport of the frozen magnetic field by the velocity field. The term $-H_{z} \times \operatorname{div} \mathrm{V}$ describes the sinking or coming to the surface caused by the sources of the transverse (horizontal) velocity field. The terms $v_{m}\left(\partial^{2} H_{z} / \partial z^{2}\right)-\nabla v_{m}(\partial \mathrm{h} / \partial z)+h \nabla v_{z}-v_{z}\left(\partial H_{z} / \partial z\right)$ may be combined into the term describing the sources of the magnetic field $H_{z}$. The term $v_{m}\left(\partial^{2} H_{z} / \partial z^{2}\right)$ describes the diffusion of the longitudinal magnetic field $H_{z}$ from under the surface. The 
term $-v_{z}\left(\partial H_{z} / \partial z\right)$ is connected with the transport of the frozen magnetic field $H_{z}$ by the vertical velocity $v_{z}$ from under the surface. The term $-v_{m}(\partial \mathbf{h} / \partial z)$ takes into consideration the changes of $v_{m}$ which cause the transformation of the transverse magnetic field $h$ into the longitudinal one during the diffusion to the surface. A similar meaning may be ascribed to the term $\mathbf{h} \nabla v_{z}$ connected with the deformation of the force lines during the transport to the surface. Practically speaking, the values included in these four expressions are unobservable and therefore we can consider only the general source of the magnetic field $\varphi$. The next detail is that we must replace $v_{m}$ with the formal diffusion coefficient $D$ which includes some processes existing simultaneously, for example the magnetic field diffusion caused by the ohmic dissipation, the diffusion of a turbulent type proposed by Leighton (1964) etc.

Now we can use the following generalized equation of the dynamical model instead of the initial one (2).

$$
\frac{\partial A}{\partial t}=\varphi-\operatorname{div}(A \mathbf{V}-D \nabla A),
$$

where $A$ is an arbitrary physical parameter (in our case $A \equiv H_{z}$ ). The problem is to reconstruct the functions $\varphi, D$ and $\mathbf{V}$ depending on the time and the coordinates on the basis of an observed time-spatial distribution of the value $A$, in other words to solve a reverse problem of the differential equations. Strictly speaking this problem is at least two times incorrect in Tikhonov's sense, because we need to compute derivatives using only numerical data surely burdened with errors, and to reconstruct the behavior of three apparently independent functions using only one function $A$. It is necessary to introduce some simplifying assumptions.

There are three ways to solve our problem.

(1) The 'global' method permits us to apply the expansions of the functions $A, \varphi$, $D, v_{x}$ and $v_{y}$ using some orthogonal basis and to replace Equation (3) with the system of linear equations for the expansion coefficients of $\varphi, D, v_{x}$ and $v_{y}$. Difficulties connected with boundaries vanish if we use a spherical surface (the whole Sun), but other obstacles appear. First it is not clear to what degree the Equation (2) used is valid in the polar regions of Sun where the observed $H_{\|}$does not correspond to the vertical component. Generally speaking this difficulty remains valid in other methods. Secondly if we want to get information about $\varphi, D, v_{x}$ and $v_{y}$ with good spatial resolution (for example $\sim 20^{\circ}$ ) then using the spherical function expansion we need about 18 harmonics, which means more than 300 coefficients for every unknown function, or a system of more than 1000 equations which surely will be very ill-conditioned.

(2) The 'continuous local' method requires us to assume the functions $A, \varphi, D, \mathrm{~V}$ to be smooth functions of coordinates in the neighborhood of the point considered and the functions $\varphi, D$ and $\mathbf{V}$ to be weakly dependent on time (practically constant) close to the moment considered. Then differentiating (3) with respect to the time we can obtain the necessary number of linear equations for the functions $\varphi, D, \mathbf{V}$ and their derivatives with respect to coordinates (no more than of the 2 nd order). In the case of 2 spatial coordinates and of anisotropic diffusion it leads to a system of 10 linear 
equations. Here we run into the incorrectness of the numerical determination of derivatives of $A$ and the solution of the linear system which as a rule is ill-conditioned. Unfortunately up to now we have had no success in working out an algorithm which provides a stable solution. It is necessary to mention that the Courant-FriedrichsLevy condition requires the time interval to be 1 solar rotation (the Mount Wilson atlas of solar magnetic fields has such a minimal step in time) so that the spatial interval for the derivative computation is of the order of $30^{\circ}$ or $40^{\circ}$. This does not permit us to use the simplest formulae of differentiation (for example 3-point formula).

(3) The 'discrete' local' method differs from the previous one in the absence of the derivative computation. Instead of this we obtain the expansions of $A$ as a function of time and of coordinates in the neighborhood of the point considered with the help of Legendre polynomials. Here we must assume the functions $\varphi, D$ and $\mathbf{V}$ to be constant during some solar rotations necessary to write down a full system of equations - that means the obtained values of $\varphi, D$ and $\mathbf{V}$ are averaged during these rotations. The Courant-Friedrichs-Levy condition is very difficult to fulfill in this case and it leads to an instability in the solution. Moreover the validity of using the Legendre polynomial derivatives is not confirmed if the order of polynomials is high.

For a first attempt we used the third method with the following parameters. The averaging interval was equal to 5 solar rotations and the coordinate step corresponded to $5^{\circ}$ (nonconformity to the Courant-Friedrichs-Levy condition). The values of $H_{\|}$ were taken from the maps of the solar magnetic fields. The computations were made for two periods: rotations 1431-1435 (September-December 1960) and 1502-1506 (December 1965-April 1966).

The instability of the solution is expressed in deviations of the same order as the terms of Equation 3 and in disordered or unreal values of velocity. Therefore the results of the computation may be considered only as accurate up to the sign. They are presented at Figures 1-6 (maps of $\varphi, D$ and divV). The positive values of $\varphi$ describe the origin of the N-polarity magnetic fields and the destruction of the S-polarity magnetic fields. The negative values of $\operatorname{div} \mathbf{V}$ correspond to the magnetic field sinking. The value orders are correspondingly of $10^{-2} \mathrm{G} / \mathrm{s}$ for $\varphi$, of $10^{3}+10^{4} \mathrm{~km}^{2} / \mathrm{s}$ for $D$ and of $10^{-3} \mathrm{~s}^{-1}$ for divV. These values seem to be real ones. Unfortunately the large averaging interval is the reason for the mixing of the dynamical picture: active regions develop and begin to decay during some months. Thus on our maps processes of increasing and decreasing of magnetic fields become superimposed and located in the same regions. This is especially noticeable for the second period. In the first period the maps reflect a complex developed system of long-living features.

The most striking fact is an appearance of negative values of the diffusion coefficient $D$. If the stable solution will confirm this fact, that means the negative diffusion coefficient is not caused by computation errors, then it shows a new unknown mechanism of magnetic field concentration by random walk opposite to Leighton's mechanism. We think that the existence of such a phenomenon is very unlikely because at first sight it contradicts the second law of thermodynamics and most probably it is caused by the incorrectness of the problem. 


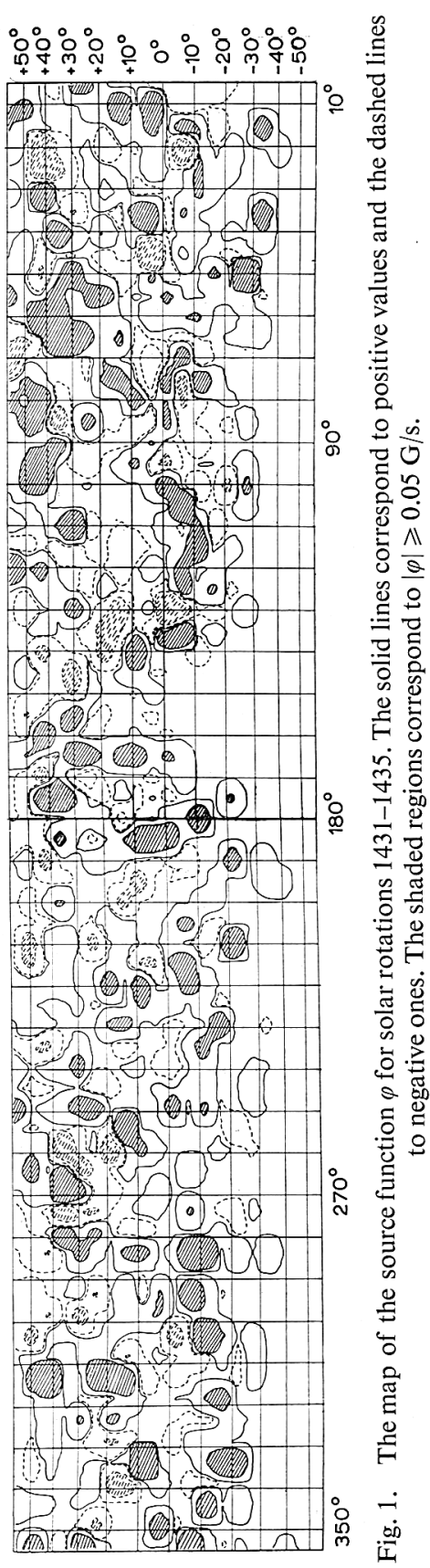

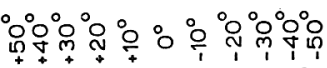

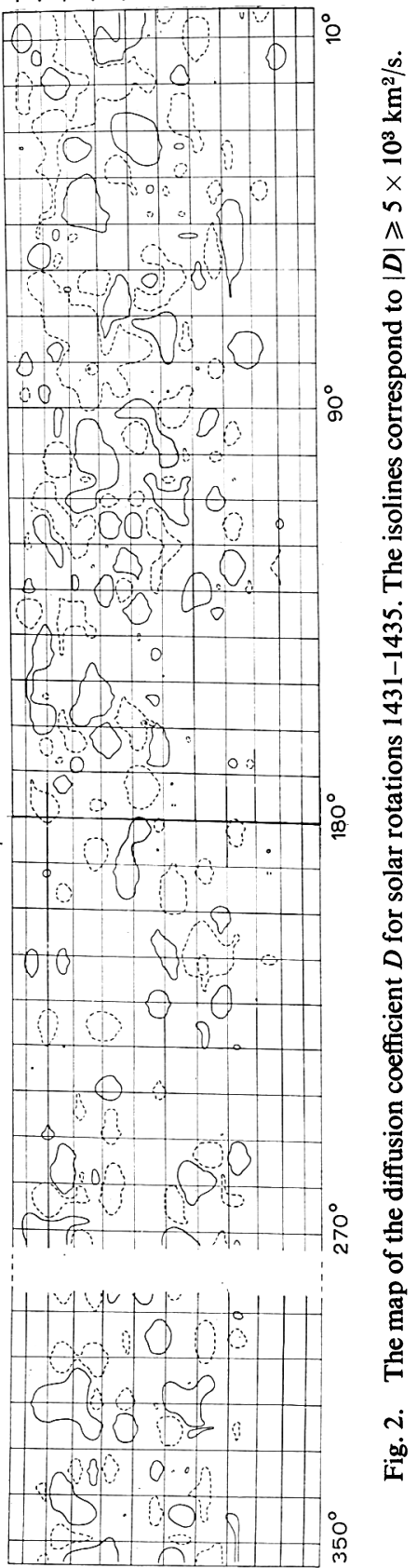




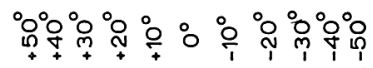

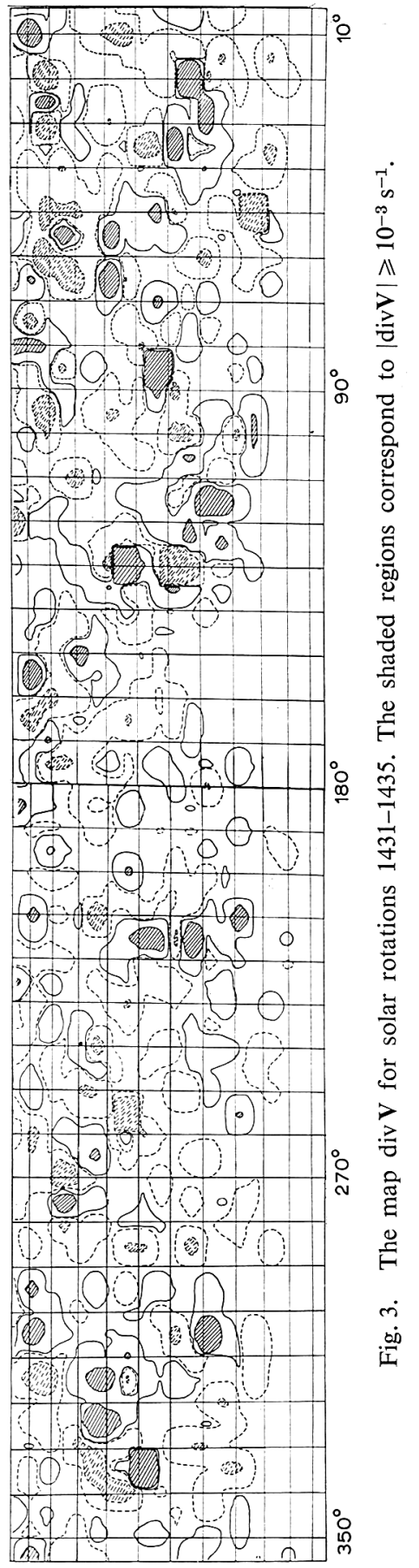

웅ำ

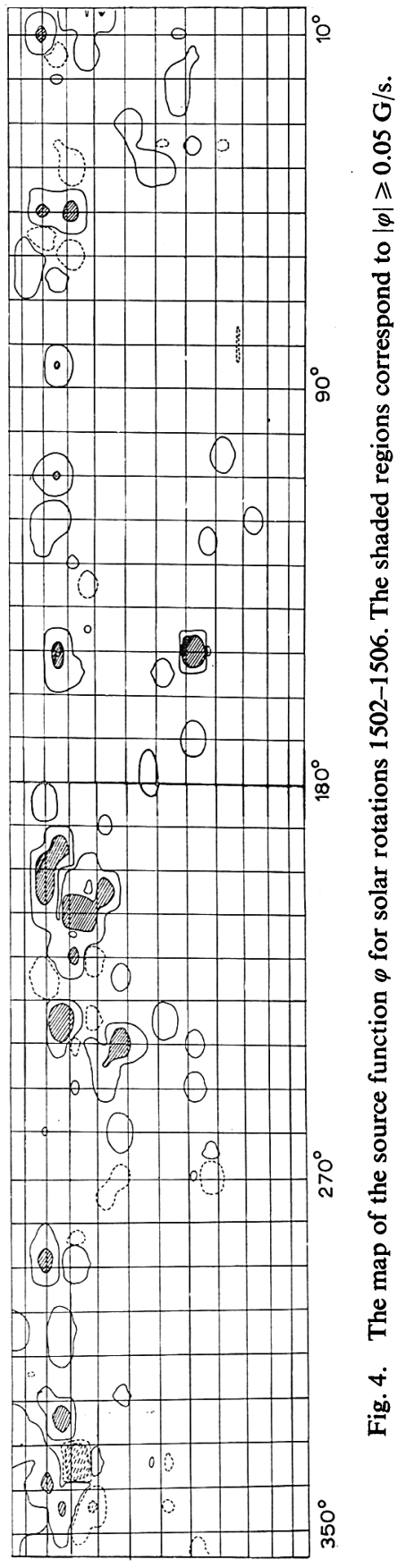




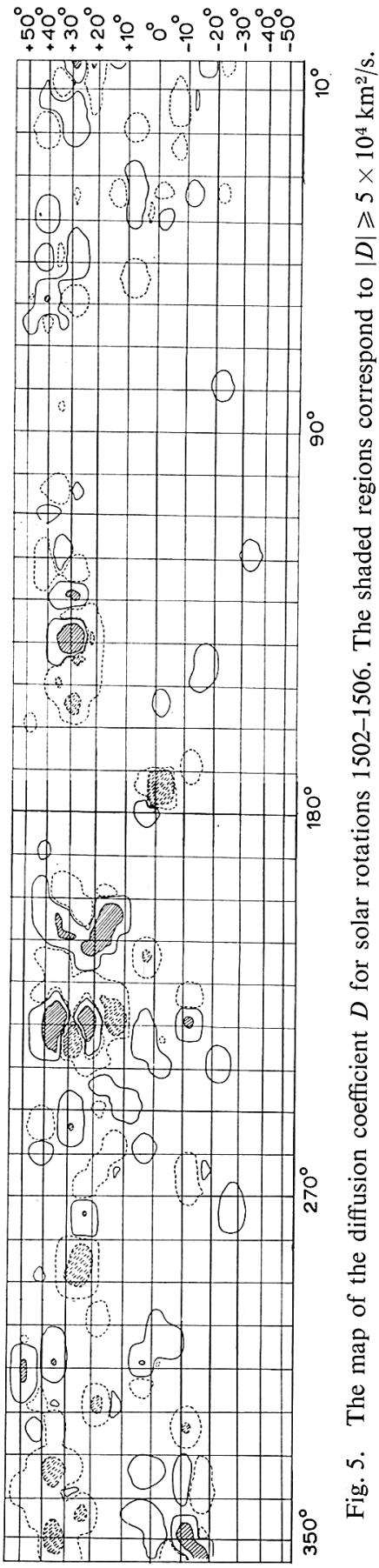

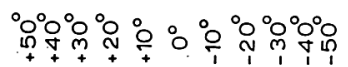

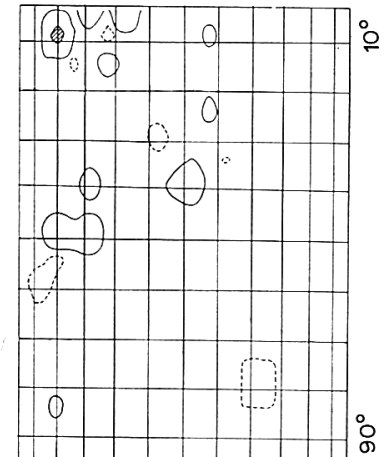

응
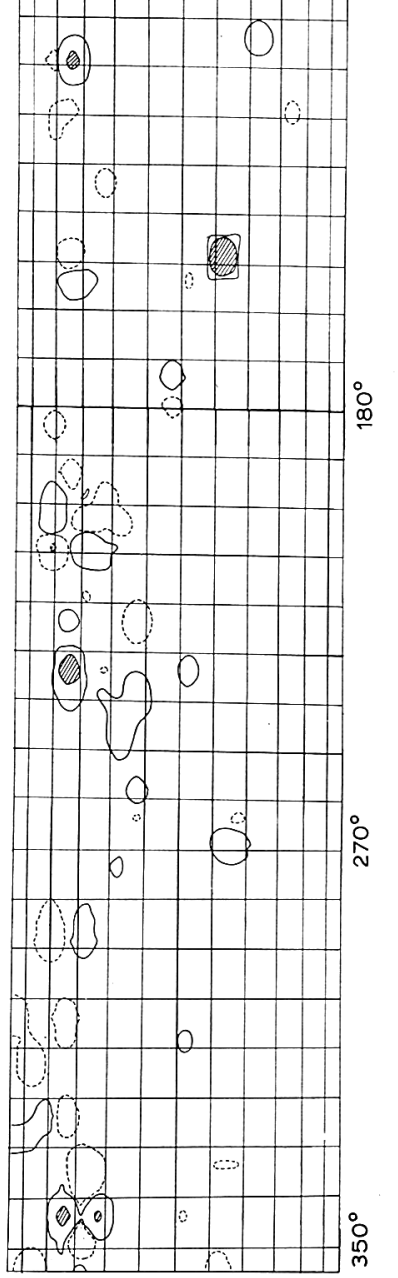
It is very interesting that the regions of ascending or descending magnetic fields (large values of $\operatorname{div} \mathbf{V}$ ) practically coincide with the regions of powerful sources of magnetic fields. The sizes of the regions are about $20^{\circ}-40^{\circ}$. Therefore the theoretical assumption about the origin of sunspots as the coming to the solar surface of the subphotospheric magnetic tubes is confirmed not for a single magnetic tube but for a whole system of tubes occupying a large region.

We intend to continue the study of the magnetic field dynamics using more perfect algorithms for the solution of incorrect problems. Perhaps our preliminary results will be confirmed and will require more detailed analysis.

\section{Acknowledgements}

The author wishes to thank Mr V. Korolev for the help in computations and Drs B. Wertlieb, S. Vainstejn, I. Krinberg, and J. Katz for valuable discussions.

\section{References}

Bumba, V. and Howard, R. F.: 1965, Astrophys. J.141, 1502.

Leighton, R. B.: 1964, Astrophys. J. 140, 1547.

\section{Discussion}

Altschuler: What was the role of the transverse magnetic field?

Kuklin: As we had no data on the transversal component of the magnetic field we included all terms depending on the transverse magnetic field (unobservable values) into the source function $\Phi$.

Gilman: I would comment that the second law of thermodynamics does not generalize to turbulent transport processes.

Kuklin: Nevertheless it is difficult to talk about discrepancies with the second law of thermodynamics loudly. 